\title{
Cryogenic Composite Disk Laser for Peak and Average Power Scaling
}

\author{
Luis E. Zapata ${ }^{1,2}$ \\ ${ }^{1}$ Research Laboratory of Electronics, Dept. of Electrical Engineering and Computer Science, \\ Massachusetts Institute of Technology, 77 Massachusetts Ave., Cambridge, MA 02139 \\ ${ }^{2}$ Center for Free-Electron Laser Science, Deutsches Elektronen Synchrotron, Notkestraße 85, D-22607 Hamburg, Germany \\ Author e-mail address: luis.zapata@mit.edu
}

\begin{abstract}
We demonstrate high gain producing $60 \mathrm{~mJ}, 200$ ps pulses at $200 \mathrm{~Hz}$ from a single 4-mm ASE limited gain-cell. A scaling paradigm utilizing a monolithic array of gain cells is proposed. OCIS codes: (140.3280) Laser amplifiers; (140.3538) Lasers pulsed; (140.3480) Lasers, diode-pumped
\end{abstract}

\section{Introduction}

Modern high energy lasers routinely produce peak intensities to drive nonlinear processes in many optical science missions e. g., for the study of interaction dynamics in extreme regimes of matter however; today's lasers lack the repetition rate to simplify data collection, for example, in the pump probe experiments at existing (and planned) FEL facilities and are woefully inadequate for practical laser driven X-ray sources, fusion drivers or particle beam accelerators, all active areas of research pursued at premier laboratories around the globe. Industrial processes for example, laser peening in the strengthening of metals, deep welding and the percussion drilling of deep holes in super-alloys -now niche markets- would flourish with the advent of practical pulsed laser sources of superior output.

Focused R\&D activities aimed at advancing the state of the art in high-average-power laser driver technology is vibrantly pursued through several European initiatives namely HiLASE, ELI and ICAN who pursue chains of cryogenic-gas cooled slab amplifiers or a coherent combination with very large numbers of arrayed fiber-lasers. Strides are reported in both of these alternative arrangements: The DIPOLE [1] project at the Appleton Rutherford laboratory reported amplification of ns pulses up to 10 Joules per pulse at $10 \mathrm{~Hz}$ repetition rate; the coherent combination of pulses ensuing from arrays of fiber-lasers [2] has been reported in proof of principle experiments at modest energies of a few $\mathrm{mJ}$ per fiber. In nanosecond lasers, 50 Joules per pulse at $10 \mathrm{~Hz}$ achieved by the MERCURY project at LLNL several years ago [3], remains the high water mark in high-energy high-repetition-rate laser drivers.

To meet the demand for high-energy high-average power laser amplifiers, under a collaborative effort between DESY and MIT we are developing a composite thin disk laser amplifier incorporating an undoped cap fashioned to eject fluorescence enabling operation at higher gain-times-aperture or $g_{0} \cdot D$ than traditional thin disks. In addition, we leverage traditional laser system thermal problems by cryogenic operation and mitigate optical problems by using a strictly relay-imaged multi-pass architecture. Finally, we propose a path forward for scaling to very high pulse energy and average power by tiling many gain-cells into a single large gain aperture.

\section{Experimental results with a $60 \mathrm{~mJ}$ output, ASE limited gain-cell}

We have begun testing composite thin disk hardware and obtained proof of principle results. Fitted with an ultrafast chirped master oscillator and grating compressor, our laboratory prototype will drive non-linear OPCPA crystals with $100 \mathrm{~mJ}, 10 \mathrm{ps}$ pulses at $200 \mathrm{~Hz}$ at DESY/CFEL when completed. A second stage to bring the pulse energy to $\sim 1 \mathrm{~J}$ is planned. The left pane in figure 1 shows the heart of the technology: a diode pumped cryogenic compositethin-disk that we estimate could surpass the performance of traditional thin-disks.

Results have shown all important performance parameters to lie within theoretical predictions for our design. In activation tests with a "control" $4.5 \mathrm{~mm}$ diameter thin disk and alternatively, a composite thin disk, the output measured 75 and 78 Watts of average power respectively and up to $220 \mathrm{~mJ}$ integrated energy under the $1 \mathrm{~ms}$ quasicw pulses with a simple muti-mode resonator.

Next, chirped pulses from a fiber mode locked oscillator were stretched in a $5 \mathrm{~m}$ long fiber and boosted to $\sim 5 \mathrm{~nJ}$ with an in-line Yb-doped fiber amplifier before injecting into a commercial $\mathrm{Yb}: \mathrm{KYW}$ regenerative amplifier (Amplitude Systemes) that output $2 \mathrm{~mJ}, 250 \mathrm{ps}$ pulses at repetition rates up to $1 \mathrm{kHz}$. The cryogenic composite thindisk was placed in a liquid nitrogen Dewar at the strict image-relay plane of a 12 pass amplifier and operated with near diffraction limited beam quality that degraded slowly up to $60 \mathrm{~mJ}$ at $200 \mathrm{~Hz}$ when a Strehl of 0.7 was measured with a HASO wavefront sensor from Imagine-optic. The Strehl dropped 0.45 at $300 \mathrm{~Hz}$ and damage ensued in the 
relay telescope optics. The bandwidth of the 200 ps pulses at full power was measured at $0.8 \mathrm{~nm}$. Technical issues in the thermo-bonding interface to the liquid nitrogen cooled heat spreader were encountered which are currently resolved.

Importantly, the storage or hold-off (without extraction) gain coefficient was measured with the uncapped disk and compared to the composite thin-disk. The right pane in figure 1 shows the data fit to our numerical ASE model yielding remarkable agreement. We will discuss our heuristic 1-Dimensional ASE design model at the conference.
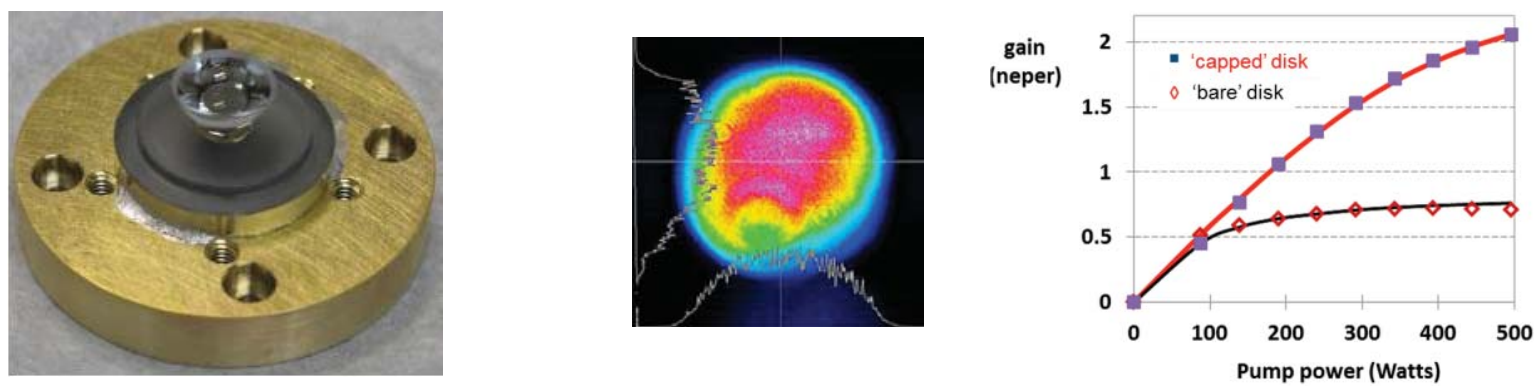

Fig. 1. A composite thin disk mounted on a heat spreader is in the left pane. The insert in the center shows the beam profile at full power and signs of the damaged optic are evident (see text). The right pane shows the measured gain hold-off for a 'bare' $1 \mathrm{~mm}$ thick, $4.5 \mathrm{~mm}$ diameter disk and the 'capped' composite disk on the left which includes an identical Yb:YAG gain sheet bonded to the thicker 'cap'. The solid curves are predictions of our numerical ASE code produced during design.

\section{The Monolithic Array of Gain Cells for scaling}

In general, the choice of amplifier geometry determines the aperture size. In bulk lasers the damage-threshold and in fibers nonlinearities respectively limit the energy that a singular aperture can produce. Complexity arises from the number of apertures that must be combined to reach the desired output. We have strived to obtain the maximum peak and average-power from a back-cooled aperture. Our variant of the thin-disk, the composite thin disk, is thermally identical to a traditional thin-disk of the same thickness however; the composite thin disk can store gain and is stiffer and resilient to deformations. Heat flows parallel to the beam towards the backplane minimizing thermo-optic as well as thermo-mechanical distortions. Cryogenic operation results in additional performance leverage as has been demonstrated, for example $[4,5]$.

In the process we invented MAGiC, the Monolithic

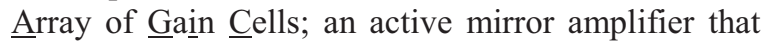
incorporates passive means for defeating transverse ASE enabling aperture scaling. We will show our method of edge-pump delivery that yields an absorbed pump distribution that is invariant in the scaling of a sturdy and large laser aperture that is a monolithically composed of neighboring gain-cells optically isolated from each other to prevent transverse ASE depleting stored gain. A tolerable diffraction loss is paid in the process. We will submit arguments to posit the MAGiC approach to scaling as a viable alternative to future high-energy high-average-power lasers.
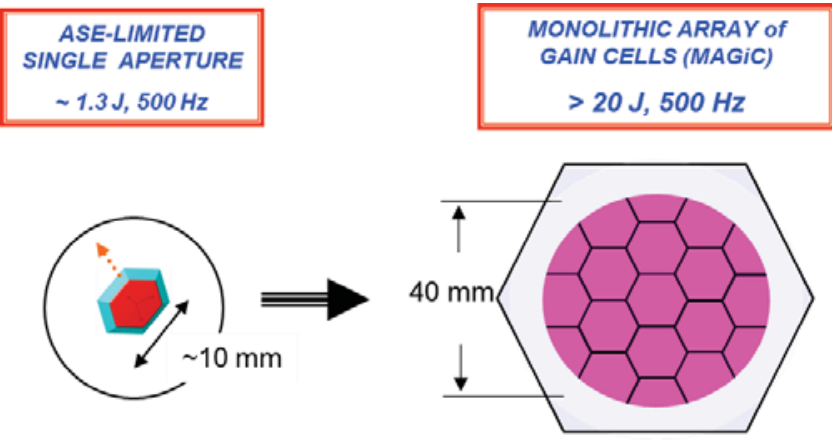

Fig. 2. Our estimate for the ASE-limited gain-cell is $\sim 10 \mathrm{~mm}$ across and delivers $\sim 1.3 \mathrm{~J}$ per pulse (left pane). Tiled crystals bonded to the bottom of an undoped, larger 'cap' can easily reach $40 \mathrm{~mm}$ in transverse dimension; the 19 groove-isolated apertures in the honeycomb pattern on the right pane would deliver $20 \mathrm{~J}$ per pulse. Aggressive liquid nitrogen impingement cooling in the backplane will enable the high repetition rate.

\section{References}

[1] S. Banerjee, K. Ertel, P. D. Mason, P.J. Phillips, M. Siebold, M. Löser, C. Hernandez-Gomez, and J. L. Collier, Optics Letters 37 [12], $2175-$ $2177(2012)$

[2] G. Moreau et al, "The future of fiber accelerators", Nature Photonics 7, 256-61 (2013)

[3] A. Bayramian, P. Armstrong, E. Ault, R. Beach, C. Bibeau, J. Caird, R. Campbell, B. Chai, J. Dawson, C. Ebbers, A. Erlandson, Y. Fei, B. Freitas, R. Kent, Z. Liao, T. Ladran, J. Menapace, B. Molander, S. Payne, N. Peterson, M. Randles, K. Schaffers, S. Sutton, J. Tassano, S. Telford, and E. Utterback, Journal of Fusion Science and Technology 52 [3], 383-387 (2007).

[4] Kyung-Han Hong, Juliet T. Gopinath, Darren Rand, Aleem M. Siddiqui, Shu-Wei Huang, Enbang Li, Benjamin J. Eggleton, John D. Hybl, Tso Yee Fan and Franz X. Kärtner, Optics Letters, Vol. 35, No. 11, June 1, 2010

[5] Darren Rand, Daniel Miller, Daniel J. Ripin, and Tso Yee Fan, Optical Materials Express, Vol. 1, No. 3, July 2011 